Cahiers de philosophie de l'université de

\title{
Différence(s) et analogie(s) : la méthode Tarde
}

\section{Anne Devarieux}

\section{OpenEdition}

Journals

Édition électronique

URL : https://journals.openedition.org/cpuc/312

DOI : $10.4000 /$ cpuc.312

ISSN : 2677-6529

\section{Éditeur}

Presses universitaires de Caen

\section{Édition imprimée}

Date de publication : 31 décembre 2017

Pagination : $35-58$

ISBN : 978-2-84133-859-7

ISSN : 1282-6545

Référence électronique

Anne Devarieux, "Différence(s) et analogie(s) : la méthode Tarde », Cahiers de philosophie de l'université de Caen [En ligne], 54 | 2017, mis en ligne le 01 février 2019, consulté le 02 février 2023. URL : http:// journals.openedition.org/cpuc/312; DOI : https://doi.org/10.4000/cpuc.312

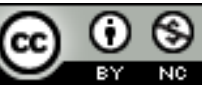

Creative Commons - Attribution - Pas d'Utilisation Commerciale 4.0 International - CC BY-NC 4.0 https://creativecommons.org/licenses/by-nc/4.0/ 


\section{Différence(s) et analogie(s) : la méthode Tarde}

$\mathrm{L}^{\mathrm{B}}$

E 28 NOVEMBRE 1862, Gabriel Tarde écrit dans son journal, il a alors 19 ans:

Je vais courant dans le monde des idées, d'autant plus libre que je ne suis pas de voie tracée, à la recherche d'analogies lointaines, de vues générales que le vague de leur énoncé soustrait à la rudesse des négations, dressant ça et là une tente nomade de touriste, sans demeure fixe, prompt à l'infidélité, non moins prompt à l'adoration et à l'extase. J'ai fini par m'imaginer implicitement que le nombre des considérations et des rapprochements pourrait suppléer à leur poids. J'explore, je découvre, je note en touriste la beauté du site et la diversité de mon admiration [...]. Ma soif de métaphysique a succédé à une soif, non plus vive, de poésie ${ }^{1}$.

À la recherche d'analogies, le métaphysicien aura-t-il jamais quitté son habit de poète, de poète géographe ${ }^{2}$ qui fixe sa tente de nomade, selon le pittoresque du site? L'analogie et l'attention scrupuleuse à la diversité, soit au pittoresque du monde en général et du monde social, semblent à elles seules dessiner le cheminement sans méthode préalable du jeune Tarde; au reste, qui a jamais considéré - objectera-t-il à Durkheim, des années après - que le progrès en science fût dû à certaines règles de méthode? Chemin sans méthode apparente, sinueux, mais non sans ordre, et dont le mouvement se déploie au gré tout à la fois de ses désirs propres, et des vues pittoresques qui s'offrent à lui, sans autre finalité semble-t-il que la « soif de métaphysique $»^{3}$ qui l'anime. Mais l'analogie et l'attention à la différence

1. Fondation nationale des sciences politiques (FNSP par la suite), Centre d'histoire de Sciences Po (CHSP par la suite), Archives d'histoire contemporaine (AHC par la suite), fonds Gabriel Tarde, GTA 64. Ce journal est encore inédit.

2. Le géographe suédois Torsten Hägerstrand à partir de la fin des années 1950 construira un modèle fondé sur ses travaux, et deviendra une référence majeure pour la sociologie des migrations en formalisant l'importance des réseaux migratoires, et en inventant la notion de «vague d'innovation».

3. FNSP, CHSP, AHC, fonds Gabriel Tarde, GTA 64. 
condensent aussi à elles seules la matière qui fournira l'aliment principal aux reproches que s'attirera la pensée d'un homme victime de son goût abusif des généralisations, assimilée à une forme originale de littérature philosophique, sans rigueur épistémologique. En témoigne exemplairement le débat qui réunit à l'École des hautes études sociales en 1903, Tarde, alors professeur au Collège de France, l'auteur des Lois de l'imitation et de la Psychologie économique, et son rival Durkheim, l'auteur des Règles de la méthode sociologique et le fondateur de L'Année sociologique, autour de la question: quelle méthode pour déterminer la spécificité du fait social ${ }^{4}$ ?

Comment dès lors entrer dans l'œuvre de Tarde, rassembler, comme il le fait lui-même dans Les lois sociales, les membra disjecta de ses pensées, où dresser nous-même notre tente de lecteur? L'œuvre de Tarde est importante: elle comporte pas moins de quinze livres, une centaine d'articles dans des revues, conférences ou essais, mais aussi des pièces de théâtre, des contes philosophiques, des fictions futurologiques, des poèmes, un journal intime, etc. Le jeune tarde est indécis, il fait du droit qui l'ennuie, rêve d'être écrivain, et décide un beau jour - car c'est une résolution - qu'il sera "philosophe avant tout, poète après " ${ }^{5}$. A-t-on là une clé? Y a-t-il un fil conducteur qui hante la pensée tardienne et lui permette a posteriori de se déployer dans des champs apparemment aussi différents que l'économie, le droit, l'histoire, la religion, la cosmologie, etc.? S'agissant du moment historique qui est le sien, de la sociologie naissante, de sa méthode et de son objet, de la frontière entre les sciences constituées et cette discipline encore balbutiante: $\mathrm{y}$ a-t-il un point de vue qui prédominerait sur l'autre? «Notre point de vue sociologique», dit souvent Tarde: quel est-il et comment l'entendre? Doit-on dire que le sociologue commande au métaphysicien ou est-ce l'inverse? La distinction des termes fait-elle seulement sens pour lui?

Si Tarde a inventé, toute invention étant une question suivie d'une réponse ${ }^{6}$, quelle est la question de Tarde derrière ses multiples interrogations? Ce lecteur boulimique, féru d'échanges vifs 7 , rumine dans la solitude à l'instar de l'inventeur de demain, sous sa «cloche à plongeur ", un problème sans actualité immédiate. Mais tout contre-courant naît du courant, et loin d'être ex nihilo, toute invention est «le croisement heureux» dans un cerveau (son «laboratoire») d'un courant d'imitation «soit avec un autre

4. Pour la mise en scène de ce débat par B. Karsenti et B. Latour: http://www.bruno-latour. $\mathrm{fr} / \mathrm{fr} /$ node/ 424 .

5. FNSP, CHSP, AHC, fonds Gabriel Tarde, GTA 65.

6. G. Tarde, Les lois sociales, Paris, Institut d'édition Sanofi-Synthélabo (Les empêcheurs de penser en rond - CEuvres de Gabriel Tarde, 4), 1999, p. 116.

7. Il déplorait l'absence d'une société de philosophes à l'image des sociétés de savants. 
qui le renforce, soit avec une perception extérieure intense qui fait paraitre sous un jour imprévu une idée reçue ${ }^{8}$ : quelle fut son invention propre?

$\mathrm{Si}$, comme il l'affirme dans sa première étude prononcée, sinon publiée, «nous portons en nous la source scellée d'une infinité d'autres passés virtuels ${ }^{9}$, nul doute que le jeune Tarde souffre de ses talents multiples, car il sait qu'il laissera derrière lui des vies mortes pour ainsi dire (le réel n'étant qu'un cas du possible); des vies jamais entièrement mortes pourtant, telle est en tout cas notre intime conviction de lecteur.

Tarde est, selon nous, rigoureusement et supérieurement individuel: méthodiquement?

Le journal, et non les seules notes manuscrites préparatoires à ses ouvrages, est à cet égard très précieux. Il y évoque sa méthode, alors qu'il n'a que 20 ans:

Janvier 1863,

L'homme d'études a toujours une méthode qui, à son insu peut-être, domine tout son travail et dirige ses efforts. Depuis quelques jours, depuis quelque temps plutôt, ma méthode se résume en une tendance continue à émettre sous une forme explicite toute idée que je sens naître implicitement en moi. Je m'habitue à écouter, d'une oreille de plus en plus affinée, sourdre au-dedans de mon esprit les idées naissantes, et aussitôt averti par ce sourd murmure comme par celui d'une source cachée, je gratte le sol et le creuse pour faire jaillir l'idée à la lumière ${ }^{10}$.

Tarde, victime de crises d'ophtalmie, est myope comme une taupe - son ophtalmie aiguisant selon lui la finesse de son ouïe -, il vit pour ainsi dire dans le noir et gratte la terre pour faire jaillir du sol ou du souterrain de son cerveau les idées qui se pressent, multiples ${ }^{11}$. Les fils de Tarde indiquent qu'il «racontait sa pensée plutôt qu'il ne l'expliquait et il la racontait comme elle lui était venue $»^{12}$. En digne héritier de Montaigne, Tarde pratique l'art consommé de la «conférence» et, comme le premier, raconte plus qu'il n'enseigne ${ }^{13}$.

8. G. Tarde, Les lois de l'imitation, $2^{\mathrm{e}}$ éd., Paris, F. Alcan, 1895, p. 47.

9. G. Tarde, Maine de Biran et l'évolutionnisme en psychologie, É. Alliez (éd.), A. Devarieux (préface), Paris, Institut d'édition Sanofi-Synthélabo (Les empêcheurs de penser en rond), 200o, p. 98.

10. FNSP, CHSP, AHC, fonds Gabriel Tarde, GTA 64.

11. Sur l'écriture de Tarde, sur la mise en forme de ses livres, nous avons des témoignages précieux: Espinas rapporte que quand Tarde composait un livre, «il commençait par écrire sur les divers éléments du sujet des morceaux courts ou longs, mais le plus souvent définitifs dans la forme, qu'il colligeait et qu'il reliait ensuite, quand il en avait un nombre suffisant, par une rédaction continue conforme au plan général» (A. Espinas, «Notice sur la vie et les œuvres de M. Gabriel de Tarde", Séances et travaux de l'Académie des sciences morales et politiques, $70^{\mathrm{e}}$ année, vol. 174 (nouvelle série 74), 1910, p. 322).

12. Gabriel Tarde: introduction et pages choisies par ses fils, suivies de poésies inédites de G. Tarde, H. Bergson (préface), Paris, L. Michaud, 1909, p. 37.

13. M. de Montaigne, Essais, liv. III, chap. II, «Du repentir», Paris, PUF (Quadrige), 2004, p. 806. 
Il ajoute, à la suite de ce même extrait du journal:

Cette méthode n'est qu'une méthode particulière; ma méthode générale est contenue dans cette parole de Montesquieu : l'esprit consiste à voir la ressemblance des choses dissemblables et la différence des choses semblables. Elle se décompose donc en deux procédés très distincts: voir le rapport des choses dissemblables, premier travail de synthèse qui unit les idées pour les féconder et les éclaire en les approchant; et voir la différence des choses semblables, travail subséquent d'analyse et de critique, plus spécial aux esprits froids et sagaces comme le précédent est plus propre aux imaginations chaudes et aux causes sympathiques. Ces deux procédés se complètent. Je m'habitue, autant qu'il est en mon humble pouvoir, d'abord à saisir les lointaines analogies et ensuite à ne point m'arrêter là, mais à discerner sainement les deux termes de la comparaison, afin de ne pas me leurrer moi-même sur sa valeur. Généraliser, individualiser; synthétiser, analyser; unir, distinguer; double instrument de l'étude sérieuse et consciencieuse qui cherche la vérité et non pas uniquement la pensée, la vérité et non la vanité. [Le malheur est que les grands généralisateurs sont de mauvais critiques, et les grands critiques de mauvais généralisateurs. Les uns se refusent à voir les analogies, par amour de l'excessive et palpable clarté; les autres, par dédain de l'observation minutieuse, se refusent à voir les différences. Tous se trompent; mais l'erreur des derniers me séduit mieux, je l'avoue.] ${ }^{14}$

Quelle est cette parole de Montesquieu? Il semble que Tarde fasse allusion à ce passage de la préface de L'esprit des lois:

J'ai d'abord examiné les hommes, et j'ai cru que, dans cette infinie diversité de lois et de mœurs, ils n'étaient pas uniquement conduits par leurs fantaisies.

J'ai posé les principes, et j'ai vu les cas particuliers s'y plier comme d'euxmêmes, les histoires de toutes les nations n'en être que les suites, et chaque loi particulière liée avec une autre loi, ou dépendre d'une autre plus générale.

Quand j'ai été rappelé à l'antiquité, j'ai cherché à en prendre l'esprit, pour ne pas regarder comme semblables des cas réellement différents, et ne pas manquer les différences de ceux qui paraissent semblables ${ }^{15}$.

La référence à Montesquieu ${ }^{16}$ est, on le voit, très éloignée de la lettre du texte, sinon de son esprit.

Dans ce même passage du journal, à sa suite, Tarde confesse son net penchant pour l'analogie, qu'Henri Bergson soulignera:

14. FNSP, CHSP, AHC, fonds Gabriel Tarde, GTA 64. Nous soulignons.

15. Montesquieu, De l'esprit des lois, in Euvres complètes, R. Caillois (éd.), Paris, Gallimard (Bibliothèque de la Pléiade; 86), 1951, t. II, p. 229. Nous soulignons.

16. Montesquieu sur lequel Durkheim a fait sa thèse latine et qu'il regarde avec Rousseau comme un précurseur de la sociologie. 
Tel est bien le double caractère de l'œuvre de Gabriel Tarde. Elle nous charme d'abord par la variété et la nouveauté des détails. Elle touche à tout ce qui nous intéresse, et elle y touche avec une baguette magique, qui fait surgir les aperçus les plus séduisants, les plus inattendus. Sociologie et psychologie, droit et politique, philosophie morale et philosophie générale, la plupart des grands problèmes ont attiré Tarde tour à tour. Ou plutôt ils l'attiraient tous ensemble; et la facilité avec laquelle cet esprit aussi souple que curieux se transportait instantanément d'un problème à un autre problème, d'une science à une autre science, explique qu'il ait pu tirer un si merveilleux parti du raisonnement par analogie, dont il avait fait le procédé essentiel de sa méthode ${ }^{17}$.

Tarde aura été un archéologue du social, tant il pratique comme les archéologues les analogies les plus lointaines afin de rattacher l'inconnu au connu. Un archéologue doublé d'un classificateur du social et d'un statisticien dont l'objet n'est autre que le dénombrement des similitudes; ce qu'il nomme "psychologie inter-mentale » (terme qu'il préfère à celui de sociologie) désigne non pas une physique sociale à la manière d'Auguste Comte, mais une paléontologie et une physiologie sociales ${ }^{18}$, la question étant de savoir si cela est le premier et dernier mot de ses investigations, et nous aurions tendance à penser qu'il en va ainsi, à condition d'élargir sensiblement le sens du mot «social ${ }^{19}$. À la façon de Maine de Biran quand il s'écrie qu'il voudrait être le théoricien de la conscience morale dont Rousseau n'aurait été, selon lui, que le poète, Tarde se sera fait le théoricien du somnambulisme social, en élargissant - comme Alexis Bertrand, éditeur de Maine de Biran et commentateur de Tarde, l'avait par ailleurs relevé ${ }^{20}$ - la psychologie à l'interpsychologie.

Quelle méthode donc? Tout est dit, nous semble-t-il, dans cette remarque du jeune Tarde citée plus haut: la méthode consiste à voir d'une part le rapport des choses dissemblables - telle est la synthèse imaginative chaude-, et à voir, d'autre part, la différence des choses semblables - telle est l'analyse

17. H. Bergson, Écrits philosophiques, Paris, PUF (Quadrige), 2011, préface aux Pages choisies de G. Tarde [1909], p. 378.

18. G. Tarde, Les lois de l'imitation, p. 112.

19. Peut-être, et sans aucun doute, aura-t-il été sensible à la notation de son maitre de jeunesse, Maine de Biran, quand celui-ci affirmait, non sans candeur, dans son journal, qu'il avait «oublié la société», tout en se décrivant lui-même comme un somnambule en société, particulièrement sensible aux considérations de Jean-Philippe François Deleuze sur le sommeil magnétique et le somnambulisme (voir le Journal de Maine de Biran, 12 juin 1815, H. Gouhier (éd.), Neuchâtel, La Baconnière, 1954, p. 86: «Mon point de vue psychologique tendait à faire de l'homme un être tout solitaire»).

20. A. Bertrand, «Un essai de cosmologie sociale: les thèses monadologiques de Gabriel Tarde», Archives d'anthropologie criminelle, t. 19, $\mathrm{n}^{\circ}$ 127-128, 1904, p. 623-66o; disponible sur Criminocorpus: https://criminocorpus.org/fr/bibliotheque/page/13775/. 
froide. Généraliser et individualiser, tenir les deux bouts, sous peine de manquer d'esprit (péché mortel), de verser dans la confusion, ou de pécher par excessive clarté: ne pas craindre l'analogie et ne pas refuser de voir la différence. Il n'y aura par conséquent pas d'analogie sans différence, pas de différence sans analogie.

Loin par conséquent qu'il faille dire avec Durkheim que si la sociologie

veut vivre, elle devra renoncer au caractère philosophique qu'elle doit à son origine et se rapprocher des réalités concrètes au moyen de recherches spéciales $[\ldots]$

Et qu'elle

n'est pas purement philosophique et [...] demande précision et objectivité21.

Comme si la philosophie elle-même n'exigeait pas la plus grande précision. L'établissement d'une science sociale engage la philosophie des faits sociaux: tandis que la première doit porter sur des faits similaires multiples, la seconde s'attache aux faits nouveaux et dissemblables. Lois de l'imitation et lois de l'invention (logique sociale), la question étant de savoir - ce que Durkheim nie - si on peut réellement dégager des lois de l'invention.

\section{Analogie: différence et répétition}

Une analogie au sens strict est un type de ressemblance. Il n'y a pas d'analogie sans ressemblance, mais toutes les ressemblances ne sont pas des analogies. Les ressemblances métaphoriques sont souvent floues et équivoques, et il y a des analogies trompeuses. Tarde n'est-il pas proche de Kant quand ce dernier affirme que la connaissance par analogie «ne signifie pas, comme on l'entend ordinairement, une ressemblance imparfaite entre deux choses, mais une ressemblance parfaite de deux rapports entre des choses tout à fait dissemblables $»^{22}$.

La science sociale suppose moins selon Tarde la notion de cause que celles de similitude et de répétition. S'il n'y a pas de science sans nombre et sans mesure, sans connaissance des ressemblances, il doit y avoir analogie

21. É. Durkheim, «La sociologie et les sciences sociales. Confrontation avec Tarde», Revue internationale de sociologie, Paris, V. Giard - E. Brière, $12^{\mathrm{e}}$ année, $\mathrm{n}^{\mathrm{o}} 1,1904$, p. 83-84. Résumé d'une conférence faite au début de l'année scolaire 1903-1904 à l'École des hautes études sociales à Paris. Reproduit dans É. Durkheim, Textes, V. Karady (éd.), Paris, Minuit (Le sens commun), 1975, vol. 1, Éléments d'une théorie sociale, p. 160-165, ici p. 161.

22. E. Kant, Prolégomènes à toute métaphysique future qui voudrait se présenter comme science, $\$ 58$, Paris, J. Vrin (Bibliothèque des textes philosophiques), 1996, p. 137. Nous soulignons. 
entre la sociologie naissante et les autres domaines de la science (la physique et la biologie notamment), non pas certes à la façon de l'évolutionnisme de Spencer, vertement critiqué ${ }^{23}$, et moins encore selon la classification d'Auguste Comte. À quoi en effet tient l'analogie entre un fait social et un phénomène naturel ? La thèse de Tarde est la suivante: la répétition en physique est analogue à la répétition organique, elle-même analogue à la répétition sociale: vibration / génération / imitation sont les trois formes de "l'universelle répétition", qu'elle opère à l'intérieur d'un même tout (et prend alors le nom d'accroissement) ou dans des touts différents (et prend le nom de série). Par conséquent, l'association est à l'imitativité ou socialité ce qu'est l'organisation à la vitalité, ce qu'est la constitution moléculaire à l'élasticité de l'éther. Matière, vie, société doivent être pensées dans leur fond commun.

Mais cette analogie, détaillée et argumentée par la lecture de très nombreux auteurs, suppose l'existence de l'essentielle différence, du dis-semblable, c'està-dire de l'invention sur laquelle la répétition est entée; qu'elles soient en effet imitatives / sociales, héréditaires / organiques ou vibratoires / physiques, toutes les répétitions viennent d'une innovation; ce qui signifie, en d'autres termes, que ce que nous nommons «ordre» provient du désordre, le «semblable» du dissemblable:

Toutes ces belles uniformités ou ces belles séries, - l'hydrogène identique à luimême dans l'infinie multitude de ses atomes dispersés parmi tous les astres du ciel, ou l'expansion de la lumière d'une étoile dans l'immensité de l'espace; le protoplasme identique à lui-même d'un bout à l'autre de l'échelle vivante, ou la suite invariable d'incalculables générations d'espèces marines depuis les temps géologiques; les racines verbales des langues indo-européennes identiques dans presque toute l'humanité civilisée, ou la transmission remarquablement fidèle des mots, de la langue cophte des anciens Égyptiens à nous, etc. - toutes ces foules innombrables de choses semblables et semblablement liées, dont nous admirons la coexistence ou la succession également harmonieuses, se rattachent à des accidents physiques, biologiques, sociaux dont le lien nous déroute ${ }^{24}$.

En lecteur admiratif et avisé de Cournot, Tarde souligne le rôle de l'accidentel, du fortuit, du contingent; mais fonder le fait social en définitive sur l'inconnu, n'est-ce pas ruiner la nécessité en science, «mettre le caprice dans les choses », «fouler aux pieds la notion de loi introduite par Comte»,

23. Un peu à la manière de Bergson dans L'évolution créatrice, dès sa première conférence sur Maine de Biran: G. Tarde, Maine de Biran et l'évolutionnisme...

24. G. Tarde, Les lois de l'imitation, p. 8. 
"ériger l'indétermination en principe » ${ }^{25}$ ? Loin qu'il fasse ainsi appel au mystère, Tarde rétorque à Durkheim la nécessité de "ne pas méconnaître au moins ce qu'on ne peut pas connaître» et met en avant notre «faculté trop peu appréciée d'affirmer au-delà de l'horizon des faits ${ }^{26}$ :

Si affirmer l'inconnu c'est nier notre ignorance, nier l'inconnu c'est ignorer deux fois ${ }^{27}$ !

Toute invention étant un possible réalisé, entre mille, parmi les possibles différents (ou «nécessaires conditionnels»), l'invention, quand elle apparaît, rend impossibles désormais la plupart de ces possibles, et possibles une foule d'autres inventions qui ne l'étaient pas auparavant.

Durkheim, s'insurge Tarde, juge l'histoire « en neptunien, non en vulcanien », c'est-à-dire qu'il néglige «le fond grimaçant des choses » ${ }^{28}$, la différence, fût-elle sous la forme de l'invention géniale, ensuite généralisée. Répétition et différence, comme dira Gilles Deleuze.

C'est par conséquent l'oubli de la différence, c'est-à-dire de l'invention, c'est-à-dire de la relation ou de la possession, nous allons le voir, qui fonde toutes les mauvaises analogies ${ }^{29}$. En effet,

[1] progrès d'une science consiste à remplacer des similitudes et des répétitions extérieures, c'est-à-dire des comparaisons de l'objet propre de cette science avec d'autres objets, par des similitudes et des répétitions intérieures, c'est-à-dire des comparaisons de cet objet avec lui-même considéré en ses exemplaires multiples et sous d'autres aspects. [...] ce n'est pas par cette comparaison, creusée et prolongée, d'un corps vivant avec un mécanisme que la biologie a progressé, c'est par la comparaison des plantes entre elles, des animaux entre eux, des corps vivants entre eux ${ }^{30}$.

L'important est là, dans la substitution aux répétitions extérieures des répétitions intérieures; comme le dit Tarde, la méthode comparative peut vérifier une hypothèse préconçue, mais si la vue de l'esprit est fausse, les résultats sont nuls:

25. Débat Durkheim-Tarde, voir note 4.

26. Ibid.

27. Ibid.

28. G. Tarde, «Questions sociales», Revue philosophique, t. 35, 1893, p. 618-638. Dans les Essais et mélanges sociologiques [Paris, A. Maloine, 1895], édition en ligne «Les classiques des sciences sociales» (université du Québec à Chicoutimi [UQAC]), p. 141, disponible à l'adresse suivante: http://classiques.uqac.ca/classiques/tarde_gabriel/essais_melanges_sociologiques/ essais_et_melanges.pdf.

29. Ainsi de celle entre biologie et sociologie par exemple, adoptée par Jacques Novicow (Conscience et volonté sociales), René Worms, ou Gustave Le Bon qui comparent tous la société à un organisme.

30. G. Tarde, Les lois sociales, p. 68. 
Ici pareillement, on a passé de généralisations hâtives fondées sur des analogies vaines et factices, grandioses et illusoires, à des généralisations appuyées sur des amas de petits faits semblables, d'une similitude relativement nette et précise ${ }^{31}$.

Il faut, par conséquent, faire descendre la sociologie des hauteurs chimériques des causes grandioses et vagues à d'infinitésimales actions réelles et précises:

Ce qu'il faut accorder aux adversaires de la théorie des causes individuelles en histoire, c'est qu'on l'a faussée en parlant de grands hommes là où il fallait parler de grandes idées, souvent apparues en de très petits hommes, et même de petites idées, d'infinitésimales innovations apportées par chacun de nous à l'œuvre commune. La vérité est que tous, ou presque tous, nous avons collaboré à ces gigantesques édifices qui nous dominent et nous protègent ${ }^{32}$.

Substituer aux oppositions extérieures des oppositions intérieures: pour l'auteur de L'opposition universelle, l'opposition en effet n'est pas un maximum de différence mais une répétition très singulière de deux choses semblables qui tendent à se détruire en vertu de leur similitude, et sont opposables en tant que forces. Le paradigme pour la sociologie encore non institutionnalisée sera l'imitation:

Que l'homme parle, prie, combatte, travaille, sculpte, peigne, versifie, il ne fait rien que tirer des exemplaires nouveaux de signes verbaux, de rites, de coups d'épée ou de fusil, de procédés industriels ou artistiques, de formes poétiques, de modèles en un mot, objets de son imitation spontanée ou obligatoire, consciente ou inconsciente, volontaire ou involontaire, intelligente ou moutonnière, sympathique ou haineuse, admirative ou envieuse, mais de son imitation toujours. C'est la pierre de touche la plus nette pour distinguer ce qui est social de ce qui est vital ${ }^{33}$.

Pas de social par conséquent sans l'exemple d'autrui : l'imitation est la mémoire sociale et son esprit, comme le nôtre, suppose jugement et volonté. C'est dire que le fait social (loin d'être une chose dont la coercivité serait une propriété intrinsèque essentielle) serait plus proprement nommé un acte social ${ }^{34}$, acte intercorporel et intermental, relevant d'une microscopie

31. Ibid., p. 52.

32. Ibid., p. 126.

33. G. Tarde, La logique sociale, Paris, Institut d'édition Sanofi-Synthélabo (Les empêcheurs de penser en rond - Euvres de Gabriel Tarde, 2), 1999, p. 63.

34. Autre point commun avec Henri Bergson qui consiste à saisir la dynamique des actes sociaux, et suppose que nous cessions de croire, à tort, que l'indistinct est en soi (sinon pour nous) indifférencié. 
sociale ou d'une psychologie intermentale, car l'apparence collective dudit fait social qu'il ne s'agit évidemment pas de nier, est en fait le résultat d'une synthèse d'ordre psychologique:

[...] chacun de nous, si orthodoxe qu'il puisse être, a sa religion à soi, et, si correct qu'il puisse être, sa langue à soi, sa morale à soi; le plus vulgaire des savants a sa science à lui, le plus routinier des administrateurs a son art administratif à lui. Et, de même qu'il a sa petite invention consciente ou inconsciente qu'il ajoute au legs séculaire des choses sociales dont il a le dépôt passager, il a aussi son rayonnement imitatif dans sa sphère plus ou moins bornée, mais qui suffit à prolonger sa trouvaille au-delà de son existence éphémère et à la recueillir pour les ouvriers futurs qui la mettront en œuvre ${ }^{35}$.

Ce qu'on nomme, par exemple, le "génie d'un peuple» est la synthèse anonyme d'originalités personnelles. À l'insuffisance des discours sur les «œuvres collectives», Tarde oppose un individualisme de méthode:

[...] au fond de on, en cherchant bien, nous ne trouverons jamais qu'un certain nombre de ils et de elles qui se sont brouillés et confondus en se multipliant. - Si simple que soit cette distinction, elle est oubliée par ceux qui, dans une institution et une œuvre sociale quelconque, contestent à l'initiative individuelle le rôle créateur, et croient dire quelque chose en professant, par exemple, que les langues et les religions sont des œuvres collectives, que les foules, les foules sans nul meneur, ont fait le grec, le sanscrit, l'hébreu, le bouddhisme, le christianisme, et qu'enfin, c'est par l'action coercitive de la collectivité sur l'individu petit ou grand, toujours modèle et asservi, nullement par l'action suggestive et contagieuse des individus d'élite sur la collectivité, que s'expliquent les formations et les transformations des sociétés. En réalité, de telles explications sont illusoires, et leurs auteurs ne s'aperçoivent pas qu'en postulant de la sorte une force collective, une similitude de millions d'hommes à la fois sous certains rapports, ils éludent la difficulté majeure, la question de savoir comment a pu avoir lieu cette assimilation générale. On y répond précisément en poussant l'analyse jusqu'où je l'ai conduite, jusqu'à la relation inter-cérébrale de deux esprits, au reflet de l'un par l'autre, et c'est seulement alors que l'on pourra s'expliquer ces unanimités partielles, ces conspirations des cours, ces communions des esprits qui, une fois formées et perpétuées par la tradition, imitation des ancêtres, exercent une pression si souvent tyrannique, encore plus souvent salutaire, sur l'individu ${ }^{36}$.

35. G. Tarde, Les lois sociales, p. 126.

36. Ibid., p. 61-62. Nous soulignons. G. Deleuze écrira: «Il est entièrement faux de réduire la sociologie de Tarde à un psycho-logisme ou même à une interpsychologie. Ce que Tarde reproche à Durkheim, c'est de se donner ce qu'il faut expliquer, "la similitude de millions d'hommes”" (Différence et répétition, Paris, PUF, 1968, p. 104-105, note 1). 
Le rapport de l'universel au particulier, aliment de la querelle entre nominalisme et réalisme, doit être compris sur le modèle du rapport entre la répétition et la variation:

Le nominalisme est la doctrine d'après laquelle les individus sont les seules réalités qui comptent; et par individus il faut entendre les êtres envisagés par leur côté différentiel. Le réalisme, à l'inverse, ne considère comme dignes d'attention et du nom de réalité, dans un individu donné, que les caractères par lesquels il ressemble à d'autres individus et tend à se reproduire dans d'autres individus semblables ${ }^{37}$.

L' "ontologique scolastique» de la sociologie de Durkheim représente pour Tarde «la plus grande erreur en sociologie» car elle consiste à croire que pour voir l'ordre, il faille sortir des détails, alors que le détail des faits renferme des adaptations saisissantes ${ }^{38}$; le génie individuel est la source de l'harmonie sociale, et l'infinitésimal, avec la différence, l'alpha et l'oméga de la sociologie. Que des réalités sociales (un code, une théologie, une grammaire) semblent nous dépasser, qu'elles s'imposent à nous individuellement par la contrainte ou la persuasion, nous oblige à nous demander comment elles se sont faites. Tarde affirme que leur origine est toute individuelle ${ }^{39}$. Il y a pourtant un réalisme social tardien mais enté sur l'attention à l'individuelle réalité. Depuis Les lois de l'imitation au moins, Tarde est convaincu que le lien social n'est ni un lien contractuel ni un échange de services, ni une contrainte imposée du dehors, qu'il n'existe pas davantage en dehors de ses manifestations individuelles, mais qu'il est un lien d'imitation. Aucun sens par conséquent à affirmer que la Société puisse exister une fois les individus écartés: «les professeurs ôtés je ne vois pas bien ce qui reste de l'Université si ce n'est un nom ${ }^{40}$.

Par conséquent, ordre, norme, loi, procèdent de l'accident, comme la répétition de la différence. Toute science peut saisir son objet par le côté similitude ou le côté différentiel, par «le verso ou le recto de la Nature»:

Ce qui importe, dans le firmament, est-ce l'accablante et majestueuse uniformité de ces gravitations répétées, de ces phases périodiques toujours les mêmes, de ces systèmes stellaires ordonnés sur des types identiques, ou bien le fondement et l'aboutissement de tout cela, les bizarres déchiquetures des nébuleuses primitives, le désordre de leurs emplacements, de leurs distances,

37. G. Tarde, Les lois de l'imitation, p. 8.

38. L'ordre est comme «l'alambic ou le moyen terme où se sublime la diversité élémentaire» (G. Tarde, "Questions sociales», in Essais et mélanges sociologiques, p. 273).

39. G. Tarde, Les lois sociales, p. 125.

40. G. Tarde, La logique sociale, p. 62. 
de leurs volumes, et l'impression singulière, d'une singularité unique en soi, essentiellement fugitive et irretrouvable, que produirait à coup sûr l'aspect du ciel sur une rétine assez vaste pour en embrasser tous les détails simultanés, pour refléter la synthèse originale, à chaque instant renouvelée, de ces répétitions banales ${ }^{41}$ ?

Le «faux air d'extériorité», l'apparence collective du fait social, témoignent d'une synthèse psychologique: loin de s'imposer par essence du dehors à l'individu sous une forme coercitive, un fait social est un acte qui a lieu de conscience à conscience, entre elles deux. À Durkheim qui entend dépasser l'ère des constructions et synthèses philosophiques, dépasser les «brillantes généralités », et donner à la sociologie son autonomie, Tarde oppose la solidarité des sciences qui rend l'autonomie ou l'égoïsme scientifique, mais encore la séparation du psychologique et du social ${ }^{42}$, illusoires.

La différence habite la répétition:

La différence est entre deux répétitions. N'est-ce pas dire inversement que la répétition aussi est entre deux différences, qu'elle nous fait passer d'un ordre de différence à un autre? Gabriel Tarde assignait ainsi le développement dialectique: la répétition comme passage d'un état des différences générales à la différence singulière, des différences extérieures à la différence interne - bref la répétition comme le différenciant de la différence ${ }^{43}$.

L'analogie suppose comme telle la différence entre ces trois formes de répétitions que Tarde ne cesse de rappeler, même s'il n'est pas toujours simple d'en assurer le fondement. Par exemple, l'évolution de l'individu est une "élicitation» par laquelle les parents sont répétés dans leur enfant, selon, nous dit-il, «je ne sais quelle action intérieure, absolument soustraite à nos yeux, foi vitale ou inspiration vitale pour ainsi dire»; de même, la conversion d'un catéchumène est la répétition en lui d'un apôtre; et l'analogie avec les choses physiques est certes plus conjecturale:

On sait cependant la vanité des efforts faits pour comprendre, par exemple, la transmission, la répétition des mouvements, soit par le contact, soit à distance, autrement qu'en supposant la communication préalable d'une force, d'une tendance cachée; et les tentatives en vue d'expliquer les combinaisons, les

41. G. Tarde, "Questions sociales», in Essais et mélanges sociologiques, p. 147.

42. On ne peut isoler par conséquent le «fait social pur» par des artifices de méthode; Durkheim pense à tort qu'il y a des tendances collectives avec leurs forces morales propres, qui dépendent de forces extérieures aux individus et nous déterminent à agir du dehors; et s'il concède qu'elles peuvent avoir des répercussions individuelles, il affirme qu'elles ne sont pas formées par les individus, mais sont indépendantes d'eux.

43. G. Deleuze, Différence et répétition, p. 104. 
formations chimiques par des groupements d'atomes vides à leur centre, dépourvus d'intérieur, n'ont jamais été plus heureuses. Concluons que, dans la nature comme dans nos sociétés, la Répétition, c'est-à-dire l'Action, va, je ne saurais trop le redire, ab interioribus ad exterior ${ }^{44}$.

Pas moins que les différences entre ces domaines, il faut souligner leur solidarité non réciproque: l'ondulation n’a pas besoin de la génération et l'imitation dépend des deux autres qui n'en dépendent pas:

Tandis que les ondes s'enchaînent, isochrones et contiguës, les êtres vivants, d'une durée assez variable, se détachent et se séparent, d'autant plus indépendants qu'ils sont plus élevés. La génération est une ondulation libre dont les ondes font monde à part. L'imitation fait mieux encore, elle s'exerce, non seulement de très loin, mais à de grands intervalles de temps. Elle établit un rapport fécond entre un inventeur et un copiste séparés par des millions d'années, entre Lycurgue et un conventionnel de Paris, entre le peintre romain, qui a peint une fresque de Pompéi et le dessinateur moderne qui s'en inspire. L’imitation est une génération à distance ${ }^{45}$.

Ainsi, de ces trois réalités que sont un ouragan, une épidémie, ou une insurrection, on doit souligner la liberté plus grande de la dernière et de la deuxième sur la première; tandis que l'imitation n'a pas besoin de traverser d'étapes, les transformations vivantes sont moins rapides que les sociales: ainsi ne s'équivalent pas le passage de la diligence à la locomotive et celui de la patte des reptiles à l'aile de l'oiseau! Voilà le naturalisme historique, conclut Tarde, relégué à sa vraie place...

De même, pour faire la part entre les similitudes, il sera bon de distinguer, en s'inspirant de l'anatomie comparée, les «analogies fonctionnelles» et les homologies: il y a des ressemblances, vitales et physiques, qui n'ont rien de social:

Le mot teotl, dans la langue des Aztecs, a beau signifier dieu aussi bien que le mot théos en grec, aucun linguiste ne verra là autre chose qu'une rencontre et, par suite, n'avouera que teotl et théos sont le même mot, mais il prouvera que bischop est le même mot qu'episcopos. La raison en est qu'un élément d'une langue ne saurait être, à un moment donné de son évolution, détaché de toutes ses transformations antérieures, ni considéré à part des autres éléments qu'il reflète et qui le reflètent; d'où il suit qu'une ressemblance constatée entre une de ses phases isolées et une des phases d'un autre vocable emprunté à une autre famille de langues et séparé de même de tout ce qui fait sa vie et sa

44. G. Tarde, Les lois de l'imitation, p. 222.

45. Ibid., p. 37. 
réalité, est un rapport factice entre deux abstractions, non un lien véritable entre deux êtres réels. Cette considération peut être généralisée ${ }^{46}$.

Il y a des rapports factices qui donnent lieu à des analogies douteuses mais aussi de vraies similitudes qui, si elles nous embarrassent, ne doivent pourtant pas être niées; les homologies étant l'équivalent des similitudes adaptatives. Si la quantité s'applique aux similitudes et que la similitude provient de la répétition - une vibration ou un mouvement périodique, une génération ou une répétition -, à de telles quantités les statistiques peuvent s'appliquer, et la science du social est possible. Enfin, ces trois principales formes de la répétition universelle pour lesquelles Tarde relève des analogies frappantes et des différences non moins instructives s'agissant de leurs relations mutuelles, sont des multiplications, ou contagions qui se répandent: qu'il s'agisse des modifications produites par une pierre tombant dans l'eau, d'un frottement d'allumettes ou de l'art de tailler le silex ${ }^{47}$, il y a des forces en mouvement auxquelles on peut assigner une direction, sinon une finalité. Car la répétition est pour la variation, pour la modulation, et elle-même procède d'une innovation; bref, la diversité est source et fin de l'unité:

Exister c'est différer. Des existences, et, par suite, des différences primordiales, c'est là l'inévitable postulat. Sur ces matériaux travaille l'effort assimilateur qui, de ces hétérogénéités brutes, se heurtant comme des couleurs simples, extrait le suc de différences toutes différentes, de variations complexes et fondues, tout intérieures. Pour aller jusqu'au bout de cette élaboration, l'assimilation intermédiaire elle-même a dû changer, comme la différenciation ${ }^{48}$.

Au commencement est l'hétérogène (affirme Tarde contre Spencer), la différence infinitésimale, bref l'invention (formation d'une nouvelle espèce végétale ou animale) sans laquelle on ne comprend pas la richesse

46. G. Tarde, Les lois de l'imitation, p. 44.

47. «Une pierre tombe dans l'eau, et la première onde produite se répète en s'élargissant jusqu'aux limites du bassin; j'allume une allumette, et la première ondulation que j'imprime à l'éther se propage en un instant dans un vaste espace. [...] Au début des sociétés, l'art de tailler le silex, de domestiquer le chien, de fabriquer un arc, plus tard de faire lever le pain, de travailler le bronze, d'extraire le fer, etc., a dû se répandre contagieusement, chaque flèche, chaque morceau de pain, chaque fibule de bronze, chaque silex taillé étant à la fois copie et modèle. Ainsi s'opère de nos jours la diffusion rayonnante des bonnes recettes de tout genre, à cette différence près que la densité croissante de la population et les progrès accomplis accélèrent prodigieusement cette extension, comme la rapidité du son est en raison de la densité du milieu» (ibid., p. 19).

48. G. Tarde, Monadologie et sociologie [1893], Paris, Institut d'édition Sanofi-Synthélabo (Les empêcheurs de penser en rond - Euvres de Gabriel Tarde, 1), 1999, p. 72. 
du divers, sa différence, son essentielle altérité. Le simple est l'hétérogène, et le complexe l'homogène:

Aussi, à cette conception courante de l'univers comme formé d'une poussière infinie d'éléments tous semblables au fond, d'où la diversité aurait jailli on ne sait comment, je me permets d'opposer ma conception particulière qui le représente comme la réalisation d'une multitude de virtualités élémentaires, chacune caractérisée et ambitieuse, chacune portant en soi son univers distinct, son univers à soi et en rêve. Car il avorte infiniment plus de projets élémentaires qu'il ne s'en développe; et, c'est entre les rêves concurrents, entre les programmes rivaux, bien plus qu'entre les êtres, que se livre la grande bataille pour la vie, éliminatrice des moins adaptés. En sorte que le sous-sol mystérieux du monde phénoménal serait tout aussi riche en diversités, mais en diversités autres, que l'étage des réalités superficielles ${ }^{49}$.

Des interférences entre ces forces-formes de la répétition, on peut dégager des lois : il y a des lois de la réfraction artistique (Platon dans Cicéron, Euripide dans Racine) comme philologique (le latin dans le milieu espagnol ou gaulois) - voir Grimm et Raynouard; il y a de même des rencontres qui aboutissent à la formation d'une nouvelle onde ou à la destruction de la rivale; il y a enfin stagnation morale quand luttent pour s'annuler la croyance et le désir; bref des interférences fâcheuses ou heureuses. Sous les répétitions, l'hétérogénéité d'originalités tumultueuses qui cherchent à se répandre, réalités physiques, vivantes ou sociales, donne le spectacle «d'ambitions infinies qui s'aiguillonnent ou se paralysent réciproquement ${ }^{50}$.

Il s'agit, en définitive, de ne pas confondre les découpages commodes de notre analyse et le mouvant du système de la différence:

Ce qu'on nomme en elles [toutes les réalités extérieures] fixité, immutabilité des lois de la nature, réalité par excellence, n'est au fond que leur impuissance d'aller plus loin dans leur voie vraiment naturelle et de se réaliser plus pleinement. Eh bien, il en est de même de ces influences fixes (momentanément fixes), d'ordre social, que la statistique découvre ou prétend découvrir; car les réalités sociales, idées et besoins, ne sont pas moins ambitieuses que les autres, et c'est en elles que se résolvent à l'analyse ces entités sociales qu'on nomme les mœurs, les institutions, la langue, la législation, la religion, les sciences, l'industrie et l'art ${ }^{51}$.

49. G. Tarde, Les lois sociales, p. 136-137.

50. G. Tarde, Les lois de l'imitation, p. 129. Bergson retiendra ce point: ce qui nous apparaît fixe ou statique, les dites lois immuables de la nature, sont des formes d'impuissance qui traduisent l'incapacité pour ces forces d'aller plus loin; il en va de même pour ce que nous nommons mœurs, institutions, langue, science, ou art.

51. Ibid. 


\section{Ambition}

Quid de cette ambition d'un monde habité par les différences et répétitions, de ce désir d'expansion (à moins que l'amour, ajoute le poète ${ }^{52}$, n'en soit l'origine)? Deleuze l'a souligné: en inscrivant les phénomènes humains dans une cosmologie, la pensée de Tarde est « une des dernières grandes philosophies de la Nature, héritière de Leibniz " ${ }^{53}$. Le monde mouvant est constitué par une série infinie de foyers vibratoires, dont chacun est source de rayonnement (vibration / génération / imitation) et l' «ambition immanente et immense qui est l'âme de l'univers " 54 est par conséquent inscrite ab initio dans la définition tardienne de la société: «la possession réciproque, sous des formes extrêmement variées, de tous par chacun » 55 .

Nous arrivons ici au cœur de la pensée tardienne, à l'analogie suprêmement audacieuse, parce qu'au fondement même de toutes les analogies, celle qui lui fait s'écrier: «Hypotheses fingo »! Elle donne lieu à une philosophie des possessions ${ }^{56}$ dont le couple élémentaire transit tout le monde sensible: la croyance et le désir. C'est elle qui permet à Tarde de substituer l'avoir à l'être, le mien au moi, l'ayant/l'eu à l'opposition stérile de l'être et du non-être, le "je désire, je crois, donc j'ai» au "fameux cogito ergo sum " ${ }^{57}$.

Telle est en effet la question fondamentale de Tarde: quelles sont les formes de possession unilatérale ou réciproque qui traversent ce monde?

Tarde ne cesse d'affirmer, quitte à s'attirer les sarcasmes de Durkheim, que nous avons, par un privilège singulier, la connaissance intime de cet élément qui n'est autre que notre conscience individuelle: par lui l'acte social est $v u$

52. «Pour dire tout le fond de ma pensée sur la source inconnue et inconnaissable des répétitions universelles, ce n'est peut-être pas seulement une ambition immense et universellement répandue qui suffit à les expliquer. Il est des jours où une autre explication, je l'avoue, me vient à la pensée. Je songe que la complaisance à se répéter indéfiniment sans jamais se lasser est un des signes de l'amour, que le propre de l'amour, dans la vie et dans l'art, est de dire et de redire toujours la même chose, de peindre et de repeindre toujours les mêmes sujets; et je me demande alors si cet univers qui semble se complaire en ses monotones répétitions ne révèlerait pas, en ses profondeurs, une dépense infinie d'amour caché, encore plus que d'ambition. Je ne puis me défendre de conjecturer que toutes choses, en dépit de leurs luttes entre elles, ont été faites, séparément, con amore, et qu'ainsi seulement s'explique leur beauté, malgré le mal et le malheur. - Mais, d'autres fois, songeant à la mort, je suis porté à justifier le pessimisme. Tout se répète et rien ne demeure: tels sont les deux caractères de notre univers, et le second dérive du premier. Pourquoi faut-il qu'il soit chimérique de concevoir même un monde parfait, à la fois stable et original, où tout demeure et rien ne se répète?...

- Mais trêve à ces rêves!» (G. Tarde, Les lois de l'imitation, chap. VIII, note p. 395).

53. G. Deleuze, Différence et répétition, p. 105.

54. G. Tarde, Les lois de l'imitation, p. 395.

55. G. Tarde, Monadologie et sociologie, p. 85.

56. Voir D. Debaise, Philosophie des possessions, Dijon, Les presses du réel, 2011.

57. G. Tarde, Monadologie et sociologie, p. 87. 
$d u$ dedans. Ce point fondamental, héritage biranien s'il en est, rapproche évidemment et par voie de conséquence Tarde de Bergson ${ }^{58}$ : à la différence des autres mondes, physique en particulier, le monde social (celui dans lequel s'opère la saisie réciproque des monades) m'est intime. Si la nature m'apparaît hermétiquement close, si je ne la saisis qu'en bloc et du dehors, ses éléments doivent pourtant se saisir entre eux: tel est le fil conducteur de Tarde semblable en cela au fil rouge de la Volonté de Schopenhauer. Si le social n'est pas mystérieux, c'est que le psychologique ne l'est pas:

Il n'y a rien de plus clair ni de plus profond à la fois que notre conscience, sorte d'estomac vitré, de ruche transparente, où se révèle à nous le secret des plus intimes opérations de la vie, qui nous émerveilleraient prodigieusement si nous n'en apercevions que du dehors les résultats, c'est-à-dire la conduite des êtres conscients. De là l'avantage ou plutôt l'obligation de demander à la psychologie, et non à la biologie avant tout, la clé de la sociologie ${ }^{59}$.

Bien que je n'agisse effectivement pas sur un atome, une jeune fille peut en revanche soigner une fleur ${ }^{60}$, et avec mes semblables, l'interaction est constante: le monde social permet la saisie réelle des monades «à nu et à vif». Là est le rapport par excellence, la possession typique dont le reste n'est qu'ébauche ou reflet; l'action possessive de monade à monade par laquelle chacune tire le monde à soi pour mieux se saisir elle-même et ainsi se transforme: tout alors est social mais ce social... est individuel. Ce point est parfaitement exprimé dans la fameuse formule des Lois de l'imitation: «Le psychologique s'explique par le social, précisément parce que le social naît du psychologique ${ }^{61}$. Ou encore selon une expression tardienne frappante:

La psychologie intermentale est une sociologie élémentaire, c'est-à-dire générale et, grâce à elle, la sociologie pourra être une science centrale et non pas seulement un nom commun donné au faisceau des sciences sociales ${ }^{62}$.

58. Voir H. Bergson, "L'intuition philosophique», in La pensée et le mouvant, in Euvres, A. Robinet (éd.), Paris, PUF, 1984, p. 1361: «Les forces qui travaillent en toutes choses, nous les sentons en nous; quelle que soit l'essence intime de ce qui est et de ce qui se fait, nous en sommes".

59. G. Tarde, La logique sociale, p. 65.

60. G. Tarde, Monadologie et sociologie, p. 90-91.

61. G. Tarde, Les lois de l'imitation, «Préface de la deuxième édition», p. VIII.

62. "Émile Durkheim et Gabriel Tarde: la sociologie et les sciences sociales. Confrontation avec Tarde », Cahiers de psychologie politique, $\mathrm{n}^{\circ}$ 10, «Documents historiques», janvier 2007, en ligne à l'adresse suivante: http://lodel.irevues.inist.fr/cahierspsychologiepolitique/index. php?id=955. Nous soulignons.

«Ce rapport d'un sujet avec un objet qui lui-même est un sujet est non pas une perception qui ne ressemble en rien à la chose perçue et qui autorise par là le sceptique idéaliste à révoquer en doute la réalité de celle-ci, mais bien la sensation d'une chose sentante, la volition d'une chose voulante, la croyance en une chose croyante, en une personne, en un 


\section{Croyance et désir}

Peut-on nier que le désir et la croyance soient des forces? Ne voit-on pas qu'avec leurs combinaisons réciproques, les passions et les desseins, ils sont les vents perpétuels des tempêtes de l'histoire, les chutes d'eau qui font tourner les moulins des politiques? Qu'est-ce qui mène et pousse le monde, sinon les croyances religieuses ou autres, les ambitions et les cupidités ${ }^{63}$ ?

Depuis sa première conférence, Tarde affirme que croyance et désir sont les deux forces indispensables à la formation et au groupement de nos sensations et sont, avec le sentir pur dont ils sont le point d'application (la sensation étant en psychologie ce qu'est la matière pour les «sciences extérieures»), les véritables éléments de l'âme, les deux catégories fondamentales. Car ces forces sont les puissances qui nous permettent de joindre, disjoindre, retenir ou repousser avec plus ou moins d'intensité, et expliquent nos combinaisons mentales apparemment les plus indécomposables ${ }^{64}$. À l'exception de ces deux forces quantitatives, tout dans l'âme est qualité pure, incapable de variation.

Parce que la croyance et le désir sont des quantités, ils peuvent être appliqués en morale, en économie, en politique, etc. Ainsi par exemple, la croyance affirmative en une idée (scientifique ou religieuse) peut croître ou diminuer, mais cette croyance affirmative est, souligne Tarde, tout autre chose que l'affirmation puis la négation de cette même idée ${ }^{65}$. De même l'augmentation puis la diminution de notre désir d'un objet est tout autre chose que notre désir puis notre répulsion de ce même objet. Croyance et désirs sont des quantités avec des signes positifs et négatifs comparables aux quantités objectives, des forces intensives comme l'effort biranien qui ne varie qu'en intensité mais reste identique à lui-même. Bref, nous affirmons, nous nions, nous désirons, nous repoussons et ces oppositions (ou «duels logiques et téléologiques») s'opèrent successivement (alternance, rythme) ou simultanément (choc, lutte et donc perte de force) pour le même objet. La conscience de l'individu est le théâtre de combats singuliers de thèses et d'antithèses, de vouloirs et de nouloirs (ne vouloir pas). Et ces oppositions sont sociales si elles se répandent par imitation.

mot, où la personne percevante se reflète et qu'elle ne saurait nier sans se nier elle-même. Cette conscience d'une conscience est l'inconcussum quid que cherchait Descartes et que le moi individuel ne lui a pu fournir» (G. Tarde, Les lois sociales, p. 55-56). Voir G. Tarde, Monadologie et sociologie, p. 90-91.

63. G. Tarde, Monadologie et sociologie, p. 50.

64. Ainsi le plaisir n'est autre que la sensation en tant que désirée; la peine, la sensation en tant que repoussée; appliqué à l'image, le désir produit le désir proprement dit. La croyance produit en effet la perception et le discernement des sens, unie avec les sensations qu'elle accouple entre elles ou sépare; exercée sur les images, elle produit le souvenir.

65. G. Tarde, Les lois sociales, p. 83. 
Dans l'ontologie vitaliste de Tarde, la conscience et la personnalité sont expliquées en termes de "monades dominantes", «éléments principaux du cerveau »; toutes les particules sont dans une relation dynamique (d'imitation et d'opposition): l'hégémonie d'une particule, i. e. l'identité des êtres et des objets, est intrinsèquement provisoire parce qu'en réalité, chaque particule «lutte pour l'assimilation et la domination", "pour la possession des particules qui l'entourent ${ }^{66}$.

Tandis que le désir fait naître les passions, les volontés et desseins, la croyance produit ce que Tarde nomme, avant Deleuze, les "percepts», mais aussi les concepts et les institutions. Le Désir est la force affective fondamentale, la Croyance la force intellectuelle. L'énergie mentale (croyance) est une "énergie de saisissement intellectuel, d'adhésion et de constriction mentale ${ }^{67}$, par laquelle l'être psychique acquiert et s'accroît, et l'énergie volontaire (désir) une énergie de tendance psychique, d'avidité mentale, par laquelle il s'extériorise et se dépense. Ainsi, les personnes se divisent selon leur manière de croire et de désirer, d'où vient leur manière de sentir: nos humeurs, nos tempéraments, nos états affectifs diffèrent, mais non pas la force qui nous pousse à croire et à désirer.

Telle est l'analogie entre le courant de désir et de foi qui traverse l'individu, et celui qui «arrose» une société: la logique sera logique de la croyance et la téléologie est téléologie du désir ${ }^{68}$. Mais les jugements et les desseins étant deux espèces du même genre ${ }^{69}$, l'objet de la logique comme de la téléologie, ce sont nos jugements qui se divisent entre eux la "somme de foi d'un homme ou d'un peuple ${ }^{70}$. La logique étudiera la répartition de la croyance et ses degrés, de même qu'elle indiquera les changements à faire pour établir l'accord, et la téléologie, devant le chaos de nos tendances ou volontés, traitera de la distribution de nos désirs.

On comprend mieux l'insistance de Tarde sur la notion d'acte: un acte de foi est au fond de tout jugement, le langage lui-même est une somme d'actes de foi en train de croître ou de diminuer ${ }^{71}$. La logique et la téléologie

66. G. Tarde, La logique sociale, p. 174.

67. G. Tarde, Les lois sociales, p. 56.

68. Contre le rationalisme, Tarde d'une certaine manière détrône le jugement, en le réduisant à une forme spéciale de la croyance.

69. G. Tarde, La logique sociale, p. 96.

70. Ibid. En ce sens, les moralistes sont des téléologistes sans le savoir.

71. Tarde développe notamment une théorie du syllogisme naturel qui se forme par le mariage de la croyance et du désir. Notre vie est remplie de syllogismes, combinaisons de la croyance et du désir qui rapprochent non pas deux affirmations ou négations, mais une assertion et une volition, ou une perception et un jugement, une volition et une image, et dont la résultante est nouvelle; les logiciens ont négligé le degré de foi que nous mettons dans nos jugements. 
(raisonnements théoriques et pratiques) traitent par conséquent de la direction de la croyance et du désir et non pas de la révélation de la vérité. La question est donc de croire assez pour désirer; de désirer assez pour croire:

Par la croyance, par le désir seulement, nous collaborons, nous nous combattons; par là seulement donc, nous nous ressemblons. [...] au fond de toutes les luttes humaines, il y a un oui ou un non, un velle ou un nolle en présence $^{72}$.

Imiter, c'est transmettre l'énergie de croyance, l'énergie de désir, courant homogène et continu qui circule identique et se communique:

Ce qui est imité c'est toujours une idée ou un vouloir, un jugement ou un dessein où s'exprime une certaine dose de croyance et désir. La contagion de l'exemple mutuel s'exerce socialement sur les croyances et désirs similaires pour les renforcer, et sur croyances et désirs contraires pour les affaiblir ${ }^{73}$.

Dans la monadologie renouvelée tardienne, l'entre-pénétration réciproque des monades implique que l'atome est un univers à lui seul, la foi et le désir tout l'être des monades, l'«instinct de sociabilité» prêté à tous les agrégats cellulaires. Qu'il s'agisse de l'adhérence moléculaire, de la nutrition pour le vivant, de la perception dans le monde intellectuel, bref de possession physique, chimique, vitale, mentale et sociale, tout être veut s'approprier les autres:

[...] et le lecteur voudra bien ne pas oublier non plus que, si la croyance et le désir, dans le sens pur et abstrait où j'entends ces deux grandes forces, ces deux seules quantités de l'âme, ont l'universalité que je leur attribue, je fais à peine une métaphore en appelant idée l'application de la force-croyance à des marques qualitatives internes sans nul rapport pourtant avec nos sensations et nos images - en appelant dessein, l'application de la force-désir à l'une de ces quasi-idées - en appelant propagande la communication d'élément à élément, non pas verbale assurément, mais spécifiquement inconnue, du quasi-dessein formé par un élément initiateur, - en appelant conversion la transformation interne d'un élément dans lequel entre, à la place de son quasi-dessein propre, celui d'autrui, etc ${ }^{74}$.

C'est la généralisation de l'individuel qui est au fond de la pensée de Tarde. Et le fondement de toutes les analogies est, non pas la spiritualisation de l'univers, mais le psychomorphisme qui supprime le hiatus entre le

72. G. Tarde, «La croyance et le désir, la possibilité de leur mesure», Revue philosophique, t. 10, 188o, p. 150-18o, ici p. 175.

73. G. Tarde, Les lois de l'imitation, p. 157.

74. G. Tarde, Monadologie et sociologie, p. 99. 
dehors et le dedans, et ce qui le rend possible à savoir la présence des deux quantités-forces mesurables, partout à l'œuvre ${ }^{75}$ :

Mais si, parmi nos états internes, autres, par hypothèse, que la sensation, il s'en trouvait de quantitativement variables, comme j'ai essayé de le montrer ailleurs, ce caractère singulier permettrait peut-être de tenter par eux la spiritualisation de l'univers. À mon avis, les deux états de l'âme, ou plutôt les deux forces de l'âme appelées croyance et désir, d'où dérivent l'affirmation et la volonté, présentent ce caractère éminent et distinctif. Par l'universalité de leur présence en tout phénomène psychologique de l'homme ou de l'animal, par l'homogénéité de leur nature d'un bout à l'autre de leur échelle immense, depuis la moindre inclination à croire et à désirer, jusqu'à la certitude et à la passion, enfin par leur mutuelle pénétration et d'autres traits de similitude non moins frappants, la croyance et le désir jouent dans le moi, à l'égard des sensations, précisément le rôle extérieur de l'espace et du temps à l'égard des éléments matériels ${ }^{76}$.

Seule une telle généralisation, permet à Tarde de rendre compte d'un univers à la fois pulvérisé et unifié:

On ne peut effectivement concevoir le monisme (cela a été dit bien des fois, je le sais) que de trois manières: soit en regardant le mouvement et la conscience, la vibration d'une cellule cérébrale, par exemple, et l'état d'esprit correspondant, comme deux faces d'un même fait, et l'on se leurre soi-même par cette réminiscence du Janus antique; soit en faisant découler la matière et l'esprit, dont on ne nie pas la nature hétérogène, d'une source commune, d'un esprit caché et inconnaissable, et l'on ne gagne à cela qu'une trinité au lieu et place d'une dualité: soit enfin en posant résolument que la matière est de l'esprit, rien de plus. Cette dernière thèse est la seule qui se comprenne et qui donne réellement la réduction demandée. Mais il y a deux façons de l'entendre. Avec les idéalistes, on peut dire que l'univers matériel, y compris les autres moi, est mien, exclusivement mien, qu'il se compose de mes états d'esprit ou de leur possibilité en tant qu'elle est affirmée par moi, c'est-à-dire en tant qu'elle est elle-même un de mes états d'esprit. Si l'on rejette cette interprétation, il ne reste plus qu'à admettre, avec les monadologistes, que tout l'univers extérieur est composé d'âmes autres que la mienne, mais au fond semblables à la mienne. En acceptant ce dernier point de vue, il se trouve qu'on enlève au précédent ses meilleurs fondements. Reconnaître qu'on ignore ce qu'est l'être en soi d'une pierre, d'un végétal, et en même temps s'obstiner à dire qu'il est, c'est logiquement insoutenable; l'idée qu'on en a, il est facile de le montrer, a pour tout contenu nos états d'esprit, et comme, abstraction faite

75. On le voit, nous sommes très loin de l'assurance avec laquelle Durkheim croit pouvoir donner à la sociologie son titre de science: où commence et où finit le fait social?

76. G. Tarde, Monadologie et sociologie, p. 45. Nous soulignons. 
de nos états d'esprit, il ne reste rien, ou l'on n'affirme qu'eux en affirmant cet X substantiel et inconnaissable, ou l'on est forcé d'avouer qu'en affirmant autre chose on n'affirme rien. Mais si l'être en soi est semblable, au fond, à notre être, n'étant plus inconnaissable, il devient affirmable ${ }^{77}$.

La généralisation de la différence est généralisation de l'individuel:

La seule manière d'expliquer la floraison des diversités exubérantes à la surface des phénomènes est d'admettre au fond des choses une foule tumultueuse d'éléments individuellement caractérisés ${ }^{78}$.

Le " point de vue sociologique ${ }^{79}$ qui conduit à admettre dans le protoplasme des éléments aux physionomies très individuelles sous leur masque uniforme, et dans l'éther des atomes caractérisés individuellement, devient compréhensible: exister c'est différer ${ }^{80}$.

De Leibniz ${ }^{81}$, Tarde retient la réduction de la matière à l'esprit et la multiplication prodigieuse des agents tout spirituels du monde, le discontinu, l'infinitésimal; de même, les actions d'hommes inventifs sont les cellules mères du corps social: au commencement sont les différences infiniment petites, et dans le petit est la source du grand, l'initiative revenant à un élément entreprenant. Un tel psychomorphisme n'est pas un idéalisme solipsiste mais une néo-monadologie:

Quelle raison avons-nous de juger à priori, ne connaissant pas le monde élémentaire, que le seul monde visible, le monde spacieux et volumineux, est le théâtre de la pensée, le siège de phénomènes variés et vivants? Comment pouvons-nous le supposer, quand nous voyons à chaque instant jaillir un être individuel, avec sa physionomie propre et rayonnante, du fond d'un ovule fécondé, du fond d'une partie de cet ovule, d'une partie qui va se circonscrivant et s'évanouissant à mesure qu'on la vise mieux, jusqu'à je ne sais quel point inimaginable? Ce point, source d'une telle différence, comment le juger lui-même indifférencié ${ }^{82}$ ?

Une telle spiritualisation du monde matériel évite l'anthropomorphisme parce que la force statique de croyance et celle dynamique de désir comportent des états inconscients (contrairement aux sensations) et sont donc

77. G. Tarde, Monadologie et sociologie, p. 43-44.

78. G. Tarde, Les lois sociales, p. 50.

79. G. Tarde, Les lois de l'imitation, p. 77.

80. «Et je comprends, dira Tarde à propos de Maine de Biran, qu'un philosophe meurt sans être revenu de l'étonnement d'exister!» (G. Tarde, Maine de Biran et l'évolutionnisme..., p. 6o).

81. G. Tarde, Monadologie et sociologie.

82. G. Tarde, Les lois sociales, p. 135 . 
objectivables $^{83}$. Est-ce à dire qu'il y ait un désir propre à l'onde? à l'espèce? à l'idée? non - ce serait une pure métaphore souligne Tarde -, mais «il faut entendre par là que les forces éparses, individuelles, inhérentes aux innombrables êtres dont se compose le milieu où ces formes se propagent, se sont donné une direction commune» ${ }^{84}$.

Avec ce couple, Tarde rejoue à sa manière l'histoire de nos deux facultés que la tradition philosophique nomme jugement et volonté (combinaisons du désir et de la croyance). Maine de Biran voulait décomposer la pensée et croyait atteindre le terme primitif avec l'effort comme dualité interne. Tarde, dont Maine de Biran fut, avec Cournot, le premier maître à penser, pense avoir atteint l'indécomposable de notre "chimie mentale» sous l'espèce de la croyance et du désir. L'erreur de Maine de Biran est d'avoir fait de l'intelligence un simple cas de la volonté, celle de Descartes et Spinoza de faire de la volonté un cas de l'intelligence; en réalité ce sont deux irréductibles et le désir a, selon Tarde, la certitude pour objet: il tend vers la croyance ${ }^{85}$. De même, Schopenhauer aurait pu universaliser la croyance - et non pas la seule Volonté qu'il aurait dû appeler correctement: désir ${ }^{86}$ !

C'est ainsi que les forces plastiques et les forces fonctionnelles de la vie, spécifiées, employées par la génération, ont leur source au-dessous du monde vivant, dans le monde physique, et que les forces moléculaires et les forces motrices de celui-ci, régies par l'ondulation, ont aussi leur source, insondable à nos physiciens, dans un monde hypophysique que les uns nomment Noumènes, les autres Énergie, les autres Inconnaissable. Énergie est le nom le plus répandu de ce mystère. Par ce terme unique on désigne une réalité qui, comme on le voit, est toujours double en ses manifestations; et cette bifurcation éternelle, qui se reproduit sous des métamorphoses surprenantes à chacun des étages superposés de la vie universelle, n'est pas le moindre des traits communs à signaler entre eux. Sous les appellations diverses de matière et de mouvement, d'organes et de fonctions, d'institutions et de progrès, cette grande distinction du statique et du dynamique, où rentre aussi celle de l'Espace et du Temps, partage en deux l'univers entier ${ }^{87}$.

83. Tarde peut affirmer que nous avons conçu la force à l'image du désir sans avoir eu l'idée de chercher dans l'objectivation de la croyance la solution des problèmes physiques et de la vie, même si implicitement nous concevons la matière à l'image de nos convictions, comme une substance cohérente et solide, et la force à l'image de nos efforts. Mais cela est une autre histoire (relative à l'origine de notre idée de force: Leibniz a imposé ici un modèle).

84. G. Tarde, Les lois de l'imitation, p. 19.

85. Ainsi l'éthique s'occupera du désir croyant.

86. Sur le vitalisme tardien, nous renvoyons à M. Lazzarato, postface de Monadologie et sociologie, "Gabriel Tarde, un vitalisme politique», p. 103.

87. G. Tarde, Les lois de l'imitation, p. 159. Nous soulignons. 


\section{Conclusion}

\section{Quel est alors le bénéfice de ce qu'il faut bien appeler la méthode Tarde?}

Par l'introduction de ce point de vue sociologique, nous aurons fait précisément ce que font toutes les autres sciences en avançant, remplacé des similitudes et des différences fausses ou vagues, en petit nombre, par d'innombrables similitudes et différences vraies et précises; ce qui est double profit pour l'artiste et le savant, et avant tout pour le philosophe qui doit, à moins de n'être rien de distinct, synthétiser les deux ${ }^{88}$.

Une telle synthèse, proprement philosophique, explique «l'individualisme supérieur ${ }^{89}$ de Tarde lui-même, la différence « différenciante » et « différenciée ${ }^{90} \mathrm{du}$ Tarde poète géographe, artiste et savant, métaphysicien-esthète ${ }^{91}$ du social. Un tel individualisme supérieur tout à la fois éclôt de la vie sociale et «se dresse pour lui résister quand elle menace de l'engloutir ${ }^{92}$.

Anne Devarieux

"Identité et subjectivité» Université de Caen Normandie

88. G. Tarde, Les lois sociales, p. 65.

89. Que l'individu soit une "petite mais populeuse société», que la relation seule le fasse exister, cela ne doit pas faire conclure à sa destitution: «Loin d'être en raison inverse de l'assimilation sociale, l'originalité individuelle est au contraire en raison directe de l'impressionnalibité aux exemples d'autrui, car, plus on concentre et on combine en soimême d'exemples différents, plus on a de chance de constituer une combinaison unique en son genre» (G. Tarde, «Sur la responsabilité morale», rapport sur la $\mathrm{XI}^{\mathrm{e}}$ question, in Actes du deuxième congrès international d'anthropologie criminelle. Biologie et sociologie (Paris, août 1889), Lyon - Paris, A. Storck - G. Masson, 189o, p. 364).

90. Dans ses «Notes philosophiques », à l'âge de 27 ans, en octobre 1870, Tarde écrit:

«La différence pure - c'est ce que j'appelle l'extériorité.

La différence différenciée, c'est ce que j'appelle l'intériorité.

La différence différenciante, c'est ce que j'appelle la supériorité»

(FNSP, CHSP, AHC, fonds Gabriel Tarde, GTA 43).

91. "C'est peu de chose, c'est chose bien passagère, une physionomie d'homme ou de femme, affinée par la vie sociale, par la vie d'imitation intense, compliquée et continue. Mais rien n'est plus important que cette nuance fugitive. Et le peintre n'a pas perdu son temps qui est parvenu à la fixer, ni le poète ou le romancier qui l'a fait revivre. Le penseur n'a pas le droit de sourire à la vue de leurs longs efforts pour saisir cette chose presque insaisissable qui n'a plus été et ne sera plus. Il n'y a pas de science de l'individuel, mais il n'y a d'art que de l'individuel. Et le savant, en songeant que la vie universelle est suspendue tout entière à la floraison de l'individualité des personnes, devrait considérer avec une modestie quelque peu jalouse le labeur de l'artiste, si lui-même, en imprimant nécessairement son cachet personnel à sa conception générale des choses, ne lui donnait toujours un prix esthétique, vraie raison d'être de sa pensée» (G. Tarde, Les lois sociales, p. 131-132).

92. G. Tarde, «L'inter-psychologie», Archives d'anthropologie criminelle, t. 19, $\mathrm{n}^{\circ}$ 127-128, 1904, p. 537-564, ici p. 551; disponible sur Criminocorpus: https://criminocorpus.org/fr/ bibliotheque/page/13689/. 